\title{
ONLINE TEACHING \\ AND DIGITAL INEQUALITIES
}

\section{A Reflection on the Practical Challenges in Teaching Logic Seminars in an Online Setting}

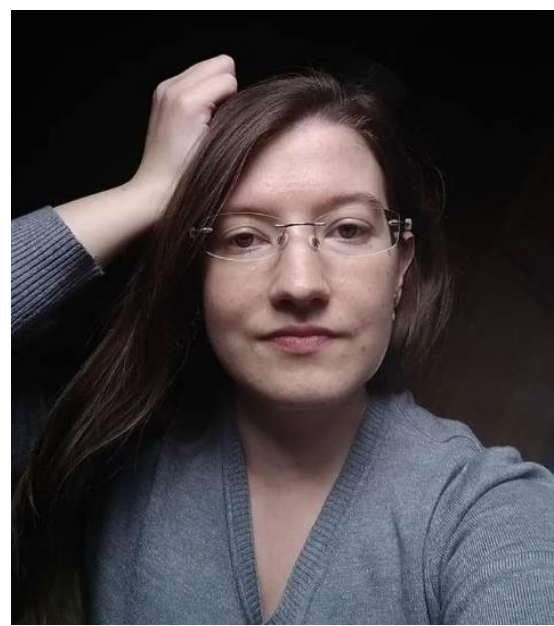

\section{Giulia Lorenzi}

PhD Candidate, Department of Philosophy, University of Warwick Correspondence: giulia.Iorenzi@warwick.ac.uk

Twitter: @GiuliaLorenzi92

Giulia Lorenzi is currently a PhD student in the Department of Philosophy at the University of Warwick funded by M4C. The aim of her $\mathrm{PhD}$ project is to explain the distinctiveness of the auditory experience of listening to music. Her areas of research mainly concern topics in auditory perception, philosophy of mind and action, and aesthetics of music. Previously, she graduated both in philosophy and in music at bachelor and master level in Italy. In 2020/2021 she taught logic seminars in the Department of Philosophy at Warwick and achieved the Associate Fellow HEA status.

\begin{abstract}
In this brief piece, I look back at the experience of teaching logic seminars in a fully online setting during the past winter, reasoning on the strategies / adopted to adapt to the situation and to mitigate difficulties emerging from digital inequalities. I highlight how, in some cases, overcoming practical difficulties generated by the online environment led to unexpected positive outcomes and how, in others, the issues persistently affected the students' experience in a way that was difficult to attenuate.
\end{abstract}

Keywords: Online Seminar; Online Teaching; New Forms of Inequalities 


\section{Online Teaching and Digital Inequalities}

With the introduction of online teaching and learning, a new type of inequality based on the possibility to access digital content emerged. In their editorial to the second issue of Learning, Media and Technology Williamson, Eynon and Potter (2020), identified several preconditions for online teaching and learning: the material availability of technological devices and their appropriateness for the intended goal, and the possession of effective digital skills. These capacities, they argue, cannot be taken for granted in our students. They are, in fact, a basis that creates an unequal distribution of learning opportunities.

While teaching online logic seminars to first year students in the Department of Philosophy at Warwick this past winter, during the third national lockdown, I realised how much my teaching practice relied on the availability to my students of specific tools and skills.

With the intention to fight the sense of isolation that many students were experiencing and to create a supportive and collaborative learning environment, I tried to promote peer-to-peer interactions and engage students in discussions at various levels. Thus, I employed in my practice general discussions, small group work, and pairs activities.

Also, the nature of the discipline I was teaching (logic) required my students to engage with the exercises and be actively involved in the seminars to develop and acquire new skills. For me, besides marking their asynchronous homework, it was essential to be able to supervise their efforts in real time and provide them feedback on their work during the seminar.
Thus, after a brief recap of the weekly topics, I used to open my seminar in Microsoft Teams asking my students to complete, under my guidance, some initial exercises in a class discussion employing the screen share function. I then used to dedicate some time to revisit together some challenging exercises that I previously identified while marking presubmitted homework. Finally, employing the breakout room function on Teams, I asked my students to collaborate in small groups or pairs to complete some sections of a worksheet that I previously made available in the file space. They could interact synchronously, giving me the chance to supervise their progress in real time. At the end of the seminar, we re-joined the main call, and I usually asked the groups to share some demonstrations, thoughts or reflections related to the exercises. I made sure to create time and space to allow them to ask questions and provide comments and feedbacks on exercises, practices, and topics we were considering.

However, these kinds of activities, that imply various forms of communication and interaction in a digital environment and the opportunity to write on an online file, are based on the opportunity to 1) use a laptop with a working microphone and camera; 2) have access to a rapid internet connection that allows fluid dialogue; 3 ) in the specific case of my seminars on logic, the ability to gain the most from the "file" session in Teams that allows synchronous interaction on a digital written worksheet.

Temporary connection issues, old or inappropriate devices (e.g. some types of phones), unexpected updates of programmes and platforms, and other circumstances clearly affected the delivery and enjoyability of the seminars over the term. This made it necessary for me to think about alternative ways to provide support to my students and navigate the online environment. 
In practical terms, the main problems I faced were related to slow internet connections, that did not allow some of my students to interact with others (and/or me) and take exercises in real time, or issues with certain versions of apps and devices that did not allow them to use specific functions. To tackle the first kind of problem, I started using channels and functions that required less data from the internet and so enabled students to communicate anyway. For example, I made ample use of the chat function that usually works even with poor internet connections, or, in other cases, I sent communications through email. In the case of temporary unavailability of the internet connection, I also planned some catch-up meetings during my office hours with single students who experienced the problem. When people could not access the space with the worksheet of weekly exercises, I sent it, in different cases before and/or during the seminars, through email. In the cases of the malfunctioning of the breakout room function that isolated some individuals from others, I often re-employed the space of the general call creating there a small group of peers.

I found collaboration with the students essential to understanding the type of problems they were experiencing and consequently find an effective way to overcome it. I also noticed that working through a practical problem generated by technological issues on a case-by-case basis allowed me to communicate more directly with single students and, consequently, probably made me sound more human and less intimidating. This mitigated the pressure that students perceived in interacting in the general discussion and with me. Indeed, when the IT issue they were experiencing was just temporary and we managed to overcome it, I noticed that some of them felt more confident and more willing to share comments and solutions when they were able to re-connect with others. So, the aspects that initially suffered more for the digital problem - the human interaction and the opportunity to contribute with their own perspective inside the session - sounded usually enhanced when the difficult circumstance was overcame. A more active participation allowed these students to both receive more feedback and support in their learning journeys, and, also, contributed to creating a more diverse and welcoming environment in the seminars.

Unfortunately, not all the difficulties were temporary: not all internet connections got better and not all devices could be replaced. In cases where problems were persistent, the most disrupted element was the opportunity to interact in real time with me and peers. In moments when isolation and feelings of loneliness were impacting on young people's mental health in a deep way, it was difficult for me to feel reassured at the idea that I had done all that I could to help them. Not being able to have a live chat on any of the elements of the module with fellow classmates or to take any exercises together with a tutor did not seem the ideal learning scenario, even when students were able to submit exercises or comments in the chat, through email or on a different website. This also affected the dynamics of the groups and the richness of the exchanges of thoughts inside the seminars that lost contributions from different voices and perspectives.

Some students also experienced difficulties in registering in and using the software (Zoxiy) used in my department for asynchronous tasks in logic. In the great majority of cases, furnishing them some further guidance with some screenshots about where to click and what to insert in some boxes was enough to make the tool available for their use. I also left different copies of the weekly worksheet in the file section of their Teams channel to allow them to engage with some exercises in case of further problems with the website. From my point of view, the use of different platforms with different 
functions and modalities of interaction sounded like an efficient method to reach different learning goals. However, I noticed that some students gave up on the use of the additional software for the exercises and found managing more than one online space (Teams, Moodle, Zoxiy, etc.) confusing for their studies. For these reasons, in addition to the problems generated by devices and connections, I did not feel confident in introducing the use of other platforms and experimenting more in the employment of technologies in my teaching practice. The fear of both creating another barrier where circumstances and times already proposed challenges, and disadvantaging individuals that already encountered major difficulties, prevented me in diversifying activities and employing other resources that I hope to try in future.

The impossibility of acting directly on the source of the problem by providing everyone the same IT resources and familiarity with them forced me as a seminar teacher to think about alternative solutions. I could get creative (in a way limited by the online data loading and devices' functions) and discover some new ways of reaching, more or less, the same pedagogical goals, and maybe, in some cases, also end up with positive unexpected outcomes. Still there were circumstances that were not completely fixable and days when I wish I could do more to mitigate certain disparities and allow all my students to get the same enjoyable experience of learning together.

\section{References}

Williamson, B., Eynon, R., \& Potter, J., (2020)

"Pandemic politics, pedagogies and practices:

digital technologies and distance education

during the coronavirus emergency", in

Learning, Media and Technology, 45:2,

https://www.tandfonline.com/doi/full/10.1080/1

$\underline{7439884.2020 .1761641}$

To cite this article: Giulia Lorenzi. 2021. “Online Teaching and Digital Inequalities: A Reflection on the Practical Challenges in Teaching Logic Seminars in an Online Setting." Journal of PGR

Pedagogic Practice, 1, 10-13. Available at: [url] 